\title{
Journal of Clinical Investigation and Studies
}

\author{
Research Article
}

\section{Ultrasound characteristics of the rotator cable in the presence of rotator cuff tears}

\author{
Fady Abdelsayed ${ }^{1}$, Hang Y $\mathbf{u}^{2}$ and Hema Choudur ${ }^{*}$ \\ ${ }^{1}$ Hamilton General Hospital, McMaster University, Hamilton ON, Canada \\ ${ }^{2}$ Michael DeGroote School of Medicine, McMaster University, Hamilton ON, Canada
}

\begin{abstract}
The rotator cable is a thick bundle of fibres cradling the distal end of the supraspinatus tendon which is known to have an avascular area that is prone to tears termed the rotator crescent. The rotator cable is thought to provide stress shielding when the rotator crescent weakens and tears. We attempted to retrospectively investigate the rotator cable in 62 patients with known rotator cuff tears diagnosed on ultrasound (U/S) imaging. We hypothesized that the rotator cable is less visible in severe tears and in older patients. In our study, we found that the cable was visualized in $89 \%$ of patients with partial-thickness tears ( $\mathrm{n}=29$ ) and in $75 \%$ of patients with incomplete full-thickness tears $(n=16)$ but in $0 \%$ of patients with complete full-thickness tears $(n=17)$. It was also found that the rotator cable visibility significantly decreased as patient age increased. Based on the results of our study, we can infer that the lack of the biomechanical stress shielding provided by the rotator cable to the rotator cuff in patients with incomplete full thickness tears predisposes the patient to a complete tear. Thus, the presence of an intact rotator cable can be used as a prognostic factor when deciding on a treatment plan for patients with varying degrees of rotator cuff tears.
\end{abstract}

\begin{abstract}
Abbreviations: FTT: Full thickness tear; iFTT: incomplete FTT; cFTT: complete FTT; REB: Research Ethics Board; MRI: Magnetic resonance imaging; NS: Not seen; NWS: Not well seen; PTT: Partial thickness tear; RC: Rotator cable; SS: Supraspinatus; U/S: Ultrasound; WS: Well seen.
\end{abstract}

\section{Introduction}

The rotator cuff comprises of the supraspinatus, infraspinatus, subscapularis, and teres minor tendons [1]. It is essential in providing both movement and stability to the glenohumeral joint [1]. At the distal confluence of the supraspinatus and infraspinatus tendons exists an avascular, crescent-shaped area known as the rotator crescent [2]. At the medial border of this crescent lies a thick fibre bundle of roughly 2.5 times thicker than the rest of the crescent, arising from the coracohumeral ligament and enveloping the supraspinatus tendon at its anterior margin [2]. This structure is termed the rotator cable and can be easily visualized using ultrasound (U/S).

The rotator cable is hypothesized to shield the rotator crescent from mechanical stress. This biomechanical role is most pronounced in older individuals as the tendons of the rotator cuff inevitably weaken with age. The rotator crescent's avascularity makes it particularly vulnerable to age-related degeneration [2]. As the crescent loses strength or even tears, the rotator cable is thought to hypertrophy to compensate [2]. This mechanism is likened to that of a suspension bridge wherein the rotator cuff serves as the bridge and the cable its support [2]. In this analogy, the stress is transferred from the rotator cuff to the rotator cable via the fibrous margins of the crescent [2].

Several anatomic studies have characterized the rotator cable through dissection or imaging [2,3,4]. However, most investigations were done exclusively on intact rotator cuffs. To our knowledge, this is the first study to evaluate the rotator cable on U/S in patients with partialthickness, incomplete full-thickness tears, and complete full-thickness tears. We hypothesize that the rotator cable is a visible structure on U/S and can be seen in patients with partial and full-thickness incomplete cuff tears but is likely absent in complete cuff tears. As the prevalence of severe tears increases with age, we also believe that the rotator cable is likely deficient in older patients, predisposing them to severe complete tears.

\section{Methods}

This study was approved by the Research Ethics Board (REB). It is a retrospective study of 62 patients, 32 men and 30 women, 18 years and older (mean age: 61.4; CI95\%: 57.8 to 65.2) from 2015 to 2016 at a single-centre with $\mathrm{U} / \mathrm{S}$ evidence of rotator cuff tears categorized as a partial-thickness tear (PTT), incomplete full-thickness tear (iFTT), or a complete full-thickness tear (cFTT).

In these patients, the supraspinatus, infraspinatus, subscapularis and teres minor tendons were examined using $\mathrm{U} / \mathrm{S}$ in the long and short axis planes to evaluate for a tear. The rotator cable could be seen during U/S examination of the supraspinatus tendon. Examples of retrieved images are shown in Figures 1 and 2. The rotator cable is seen as an echogenic band separate from the supraspinatus tendon along the medial aspect of the distal tendon, adjacent to the critical zone (avascular area of the distal tendon). A single musculoskeletal radiologist reviewed each U/S image. The rotator cable was categorized as well seen (WS), not well seen (NWS), or not seen (NS). Relationship was determined by $\chi^{2}$ test and clinical significance was determined by $p<0.05$. The mean ages of

*Correspondence to: Hema Choudur, Professor of Radiology, Head: Division of MSK Imaging, McMaster University, Hamilton General Hospital, 237 Barton St. E, Hamilton, On L9L 2X2, Canada, Tel: 905-527-4322 ext 46521; E-mail: hnalinic@yahoo.com; choudur@hhsc.ca

Received: June 15, 2018; Accepted: June 21, 2018; Published: June 29, 2018 


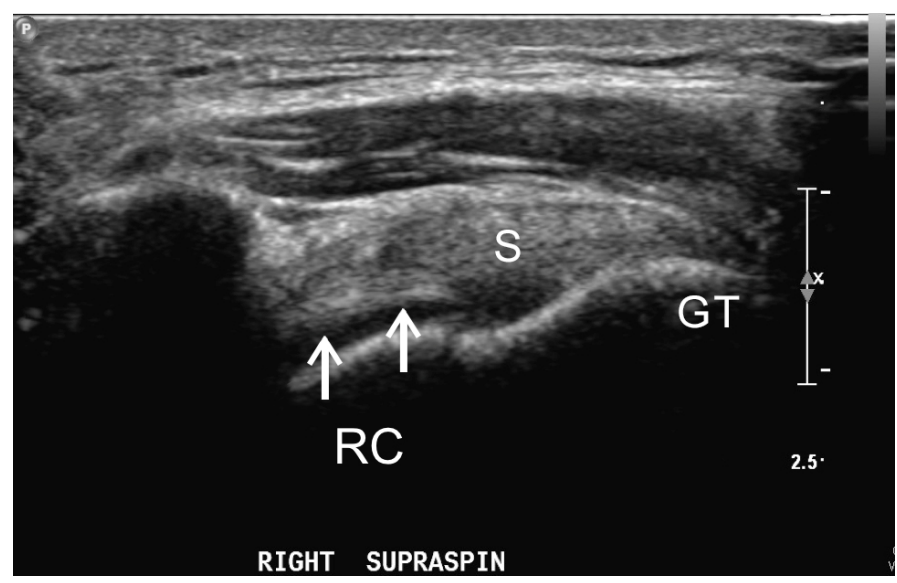

Figure 1. Long axis Supraspinatus (S) tendon. The rotator cable (RC) is well seen in a patient with a partial thickness articular aspect and footprint tear of the supraspinatus tendon. The tear is seen as a small hypoechoic area along the undersurface of the distal tendon. GT $=$ Greater tuberosity of the humerus

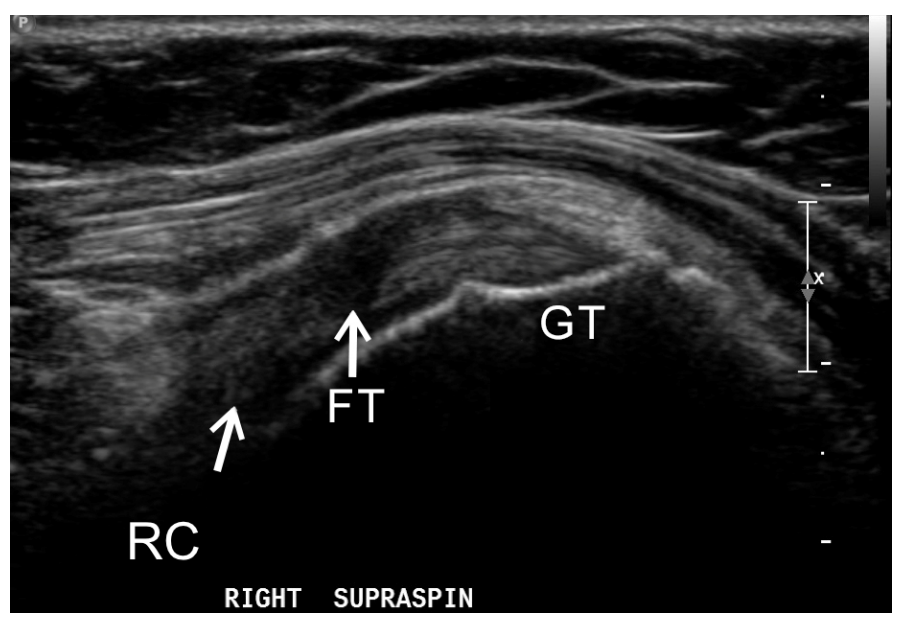

Figure 2. Long axis SS (Supraspinatus) tendon. The rotator cable (RC) is not well seen in a patient with a full thickness (FT) tear of the supraspinatus tendon at the critical zone. $\mathrm{GT}=$ Greater tuberosity of the humerus

patients with a cFTT, iFTT, and PTT and with a WS, NWS, and NS rotator cable were also tabulated and compared using ANOVA.

\section{Results}

From Table 1, the rotator cable was visible in $61 \%(n=38)$ of patients and not seen in $39 \%(n=24)$ of patients. It was well seen in $32 \%(n=20)$, not well seen in $29 \%(n=18)$ and not seen in $39 \%(n=24)$. Further, the cable was not seen in $100 \%$ of patients with complete full-thickness tears $(n=17)$. In contrast, it was visualized in $89 \%$ of patients with partial-thickness tears $(\mathrm{n}=29)$ and in $75 \%$ of incomplete full-thickness tears $(n=16)$. The $\chi^{2}$ was calculated to be 38.1 with 4 degrees of freedom corresponding with a $\mathrm{p}<<0.001$. Thus, the correlation between rotator cable visibility and severity of cuff tears is clinically significant.

The results also show that the severity of rotator cuff tears increases with age (Table 2). Patients with complete full-thickness rotator cuff tears had a mean age of 68.5 years versus 66.2 years for incomplete fullthickness tears and 55.9 years for partial-thickness tears. The rotator cable was also found to be better seen in younger patients. Those with WS cables had an average age of 49.9 years, versus NWS at 62.9 and NS at 70.4. ANOVA of this data set yields an f-ratio value of 12.77 and a p-value of .000025 indicating clinical significance.

\section{Discussion}

Rotator cuff tears are common and are thought to affect anywhere from $5-40 \%$ of adults [1]. While the majority are asymptomatic, they can also negatively affect the strength and stability of the shoulder and are a common cause of shoulder pain and disability among adults [1]. The prevalence of rotator cuff tears is age-related. Due to cumulating mechanical stress and increasing tendon laxity with age, the rotator cuff is more prone to tearing in older individuals [1]. The most common area of injury is termed the "critical zone," and includes the portion of the supraspinatus tendon that houses the rotator crescent [5]. Progressive injury to this area actually changes the dynamics of the rotator cuff causing the rotator cable to play a more prominent role in shoulder strength [2,5]. Burkhart, Esch, and Jolson (1993) characterized rotator cuffs as either crescent-dominant, or cable-dominant depending on whether there is stress shielding by the rotator cable. Crescentdominant cuffs were hypothesized by the authors to eventually evolve into cable-dominant cuffs with progressive biomechanical damage and tearing [2].

The rotator cable is well-known to anatomists, radiologists and orthopedic surgeons, and its structure has previously been extensively characterized with cadaveric dissections, histopathology, and imaging $[2-4,6]$. Overtime, it has been made increasingly clear that the rotator cable carries with it several clinical implications in the management of rotator cuff tears. The stress shielding effects of the rotator cable may allow patients to be asymptomatic when an intact cable compensates for weakness caused by smaller rotator cuff tears $[2,3]$. However, larger tears and those that involve the rotator cable may manifest as substantial loss of supraspinatus strength and consequently disability [2,3].

There are a few limitations to our study. First, our study is retrospective and contains a relatively small sample size of 62 . Additionally, the U/S imaging were performed by multiple technicians. As U/S is operator-dependent, the ability to visualize the rotator cable could have been influenced by varying operator expertise. Similarly, patients with rotator cuff tears usually have more limited range of motion which may also impact visibility on U/S. Finally, there was no comparison of cable visibility in patients with intact rotator cuffs in our study.

Our current study can develop the framework for a future prospective study that incorporates a larger sample size. Several adjustments can be made to the methodology of this study to further improve the quality of evidence. As U/S is operator-dependent, having only one technologist perform all the U/S scans will reduce variability

Table 1. Visualization of the rotator cable relating to severity of rotator cuff tear. cFTT complete full-thickness tear, iFTT incomplete full-thickness tear PTT partial-thickness tear, WS well seen, NWS not well seen, NS not seen

\begin{tabular}{|c|c|c|c|c|}
\hline Tear & Total=62 & WS & NWS & NS \\
\hline cFTT & $27 \%(17)$ & 0 & 0 & 17 \\
\hline iFTT & $26 \%(16)$ & 6 & 6 & 4 \\
\hline PTT & $47 \%(29)$ & 14 & 12 & 3 \\
\hline
\end{tabular}

Table 2. Rotator cuff tear and rotator cable visibility with respect to patient age. cFTT complete full-thickness tear, iFTT incomplete full-thickness tear PTT partial-thickness tear, WS well seen, NWS not well seen, NS not seen

\begin{tabular}{|c|c|c|c|c|c|}
\hline \multicolumn{3}{|c|}{ Rotator Cuff Tear } & \multicolumn{3}{c|}{ Rotator Cable } \\
\hline \multicolumn{2}{|c|}{ Mean Age (years; CI 95\%) } & \multicolumn{3}{c|}{ Mean Age (years; CI 95\%) } \\
\hline cFTT & iFTT & PTT & WS & NWS & NS \\
\hline 68.5 & 66.2 & 55.9 & 49.9 & 62.9 & 70.4 \\
\hline$(62.5-72.6)$ & $(58.3-74.1)$ & $(50.7-61.1)$ & $(43.4-56.3)$ & $(56.6-69.2)$ & $(65.5-75.3)$ \\
\hline
\end{tabular}


within the data. The images can be read by at least two blinded radiologists in order to measure inter-reader reliability. Finally, MRI may also be used to characterize the rotator cable alongside U/S and a comparison can be made between the two modalities. There is currently limited peer review research investigating the rotator cable using MRI [4]. Although U/S is equivalent to MRI in determining a rotator cuff tear, MRI still provides superior soft tissue imaging and is not operatordependent [7].

In summary, the rotator cable is readily identifiable in shoulder $\mathrm{U} / \mathrm{S}$ especially in younger patients and those with incomplete fullthickness and partial-thickness tears. It was seen that less severe cuff tears correlated with a higher likelihood of an intact rotator cable. We believe that the identification of the rotator cable should be part of the routine shoulder $\mathrm{U} / \mathrm{S}$. The presence of the rotator cable can be used a prognostic factor when deciding on a treatment plan for patients with rotator cuff tears. Patients with a visualized intact cable are likely better able to compensate for their cuff injury and likely require more conservative management for their pathology.

\section{Conflicts of interest}

Each author provided equal work on this manuscript. The authors note there are no competing conflict of interest.

\section{Acknowledgements}

None

\section{References}

1. Wani Z, Abdulla M, Habeebullah A, Kalogriantis S (2016) Rotator cuff tears: Review of epidemiology, clinical assessment and operative treatment. Trauma. 18:190-204.

2. Burkhart SS, Esch JC, Jolson RS (1993) The rotator crescent and rotator cable: an anatomic description of the shoulder's "suspension bridge." Arthroscopy. 9:611-616. [Crossref]

3. Morag Y, Jacobson JA, Lucas D, Miller B, Brigido MK (2006) US Appearance of the Rotator Cable with Histologic Correlation: Preliminary Results. Radiology. 241:485491. [Crossref]

4. Gyftopoulos S, Bencardino J, Nevsky G, Hall G, Soofi Y, et al. (2013) Rotator Cable: MRI Study of Its Appearance in the Intact Rotator Cuff With Anatomic and Histologic Correlation. AJR Am J Roentgenol. 200:1101-1105. [Crossref]

5. Codman EA, Akerson IB (1931) THE PATHOLOGY ASSOCIATED WITH RUPTURE OF THE SUPRASPINATUS TENDON. Ann Surg. 93:348-359.

6. Bureau NJ, Blain-Paré E, Tétreault P, Rouleau DM, Hagemeister N (2016) Sonographic Visualization of the Rotator Cable in Patients With Symptomatic Full-Thickness Rotator Cuff Tears: Correlation With Tear Size, Muscular Fatty Infiltration and Atrophy, and Functional Outcome. J Ultrasound Med. 35:1899-1905. [Crossref]

7. Singh JP (2012) Shoulder ultrasound: What you need to know. Indian J Radiol Imaging 22:284-292. [Crossref]

Copyright: (C2018 Abdelsayed F. This is an open-access article distributed under the terms of the Creative Commons Attribution License, which permits unrestricted use, distribution, and reproduction in any medium, provided the original author and source are credited. 